\title{
Spherical Aberration of Point Spread Function with Asymmetric Pupil Mask
}

\author{
Naresh Kumar Reddy Andra ${ }^{1,2,3}$ and Karuna Sagar Dasari ${ }^{3}$ \\ ${ }^{1}$ Science and Research Laboratory of Automated Systems of Science Researches, Samara National Research University, \\ Moskovskoye Shosse 34, Samara 443086, Russia \\ ${ }^{2}$ Department of Physics (HÆS), CMR Institute of Technology, Medchal Road, Kandlakoya, Hyderabad, Telangana 501 401, India \\ ${ }^{3}$ Optics Research Group, Department of Physics, University College of Science, Osmania University, No. 49, Hyderabad, \\ Telangana 500007, India \\ Correspondence should be addressed to Naresh Kumar Reddy Andra; naarereddy@gmail.com
}

Received 31 July 2016; Accepted 9 November 2016

Academic Editor: Paramasivam Senthilkumaran

Copyright (C) 2016 N. K. R. Andra and K. S. Dasari. This is an open access article distributed under the Creative Commons Attribution License, which permits unrestricted use, distribution, and reproduction in any medium, provided the original work is properly cited.

\begin{abstract}
Point spread function underneath spherical wave aberration with antiphase apodization has been obtained by one-dimensional pupil mask functions. In the presence of spherical aberration, suppression of optical side-lobes has increased on one side of the point spread function with the width of the periphery strips within the pupil mask. On introducing wave aberration effect, there exists dependence of the lateral resolution of central peak of the asymmetric point spread function on the amount of amplitude masking. However, the magnitude of intensity of central peak is originated be to amplified by the highest degree of amplitude and phase masking. Additionally, for aberrated asymmetric PSF, FWHM increases and it further decreases with the control parameters of amplitude and phase mask. The magnitude of this corollary can quantify the super resolution of diffracted structures under spherical aberration.
\end{abstract}

\section{Introduction}

Asymmetric apodization is the designed modification of the impulse response of an optical system such that simultaneous elimination of secondary maxima and narrowing the central peak in the field of diffraction. This method can change the performance of an optical imaging system by redistributing the light flux in the image plane. There has been a certain number of investigations involving asymmetric apodization for different considerations, that is, improvement of side-lobe suppression $[1,2]$, application of asymmetric apodization to linear arrays and circular arrays $[3,4]$ to improve the axial resolution of the optical system $[5,6]$, reduction of Sparrow resolution limit with incoherent illumination [7] to achieve axial and lateral resolution in confocal imaging [8] and to detect the extra solar planets [9], and improvements of dispersion of chirped fiber gratings [10]. In these studies the optical system has asymmetrically apodized. It is clear that by employing asymmetric apodization we obtained low sidelobes and sharp main peaks on one side of the PSF at the cost of improved side-lobes and wider main peaks on counter side.

It is well known that diffractive aberrations are caused by deviations from geometrical optics due to the wave nature of light. These effects place the limit on the optical system imaging performance. The third-order Gaussian theory describes that primary monochromatic aberrations like spherical aberrations arise due to geometrical deviations and may also result from the pupil functions. The spherical aberration can also be explained by saying that the deviation of the principal surface from its ideal shape causes the focal length to slightly vary with the radius of zone of the aperture. There has been a significant number of studies involving diffractive monochromatic aberrations with different aperture systems and techniques [11-13].

It is clear that, through suitable apodization technique in the exit pupil of optical system, the diffractive object may 
achieve high resolution over a confined region in the image plane of an optical system in the presence of Seidel aberration effect. This is the origin for the current investigation. Based on the extensive study made on the system of apodization, we came into conclusion that asymmetric apodization could be the solution for aberrated optical imaging system to achieve the super resolution. This technique is effective in improving the axial and lateral resolution. In this study we investigated cylindrical optical elements or slit functions with asymmetric apodization employed to redistribute the light energy in the focal plane under geometrical aberration. The study has a wide range of potential applications such as confocal imaging, medical optics, laser beam profiling, atmospheric science, and design of antenna arrays in communication engineering. In this paper work, we obtained asymmetric point spread function of one-dimensional asymmetric pupil mask configured analytically in the structure of two periphery fine strips of certain width subjected to phase masking and a central cylindrical region of the aperture is shaded with amplitude mask. Here we obtained first side-lobe intensities, central peak positions, and FWHM values for various amounts of amplitude and phase apodization.

\section{Theory and Formulation}

Within the diffraction wave theory, the amplitude impulse reaction of spherical aberration strained optical imaging system is the Fourier transform of the asymmetric pupil mask function which consists of three regions having homogeneous amplitude transmittance, namely, two fine edge strips with reverse phase transmittance of the forms $\exp (-i \pi / 2)$ and $\exp (i \pi / 2)$ and zero phase transmittance for the central cylindrical amplitude mask of the one-dimensional optical element. Because of exceptionally deep reduction ability and constant working angles throughout the regions of considered edge elements, we consider the asymmetric phase functions. Thus, we consider complex pupil mask with real amplitude transmittance at the central region and complex conjugated outer edge elements. In this case, the resultant complex light amplitude distribution $I(u)$ in the focal plane is the sum of the diffraction amplitude contributing to the central cylindrical region of aperture system of width $(1-2 a)$ and diffraction amplitudes contributing to the narrow left and right periphery strips with opposite phase transmittances $-i$ and $i$ given by

$$
\begin{gathered}
\text { Left edge phase mask }=-i, \quad-\frac{1}{2} \leq r<-\frac{1}{2}+a \\
\text { amplitude mask at center } \quad-\frac{1}{2}+a \leq r \leq \frac{1}{2}-a \\
\text { Right edge phase mask }=+i \quad \frac{1}{2}-a<r \leq \frac{1}{2} .
\end{gathered}
$$

The diffraction field amplitude contributing to the left periphery fine strip is as follows:

$$
G_{p}(u)=\int_{-1 / 2}^{-1 / 2+a}-i \exp (i 2 u r) d r .
$$

On introducing wave aberration, such as primary spherical aberration, the diffraction amplitude contributing to central cylindrical amplitude masking zone of a pupil function of width $(1-2 a)$ is as follows:

$$
\begin{aligned}
& G_{q}(u)=\int_{-1 / 2+a}^{1 / 2-a}\left(1-4 \beta r^{2}+4 \beta r^{4}\right) \exp \left[\frac{-i S r^{4}}{4}\right] \\
& \cdot \exp (i 2 u r) d r .
\end{aligned}
$$

The diffraction field amplitude contributing to the right periphery fine strip is as follows:

$$
G_{r}(u)=\int_{1 / 2-a}^{1 / 2} i \exp (i 2 u r) d r .
$$

Therefore, the total field amplitude on the image plane of the optical imaging system with asymmetric pupil mask is

$$
\begin{aligned}
G(u)= & \int_{-1 / 2}^{-1 / 2+a}-i \exp (i 2 u r) d r \\
& +\int_{-1 / 2+a}^{1 / 2-a} \exp \left[\frac{-i S r^{2}}{2}\right] \exp (i 2 u r) d r \\
& +\int_{1 / 2-a}^{1 / 2} i \exp (i 2 u r) d r
\end{aligned}
$$

where $u=(2 \pi / \lambda) \sin \theta, r$ is the coordinate in the pupil plane, $u$ is the reduced dimensionless diffraction optical coordinate, $\lambda$ is the wavelength of the stimulated light radiation, and $S$ is the spherical aberration control parameter of the optical imaging system. " $a$ " and $\beta$ are phase (asymmetric) apodization and amplitude apodization control parameters, respectively. Impact of primary spherical aberration on resultant intensity PSF, $B(u)$ which is the actual quantifiable quantity, can be obtained by taking square modulus of (5). Accordingly,

$$
B(u)=|G(u)|^{2} .
$$

\section{Results and Discussion}

The results of investigations on the effects of spherical aberration on the light flux distributions in the image plane of an optical imaging system with asymmetric pupil functions have been obtained from (6) as a function of dimensionless optical coordinate " $u$ " varying from -15 to +25 by employing an iterative method of numerical integration. Gaussian quadrature method [14] holds the most important properties and accuracy in finding the positions and intensities of primary and secondary maxima on either side of PSF under primary spherical aberration, though results are reported on one side, the PSF only, in which side-lobes are suppressed to a great extent and central peak is found to be sharp. This is the right half of the entire pattern. Our main attention has been drawn on central peak position and its resolution, optical side-lobe intensities for various amounts of spherical aberration, and amplitude-phase apodization. These parameters are enough for judging the imaging performance and efficiency of asymmetric pupil mask of spherical aberration strained optical 


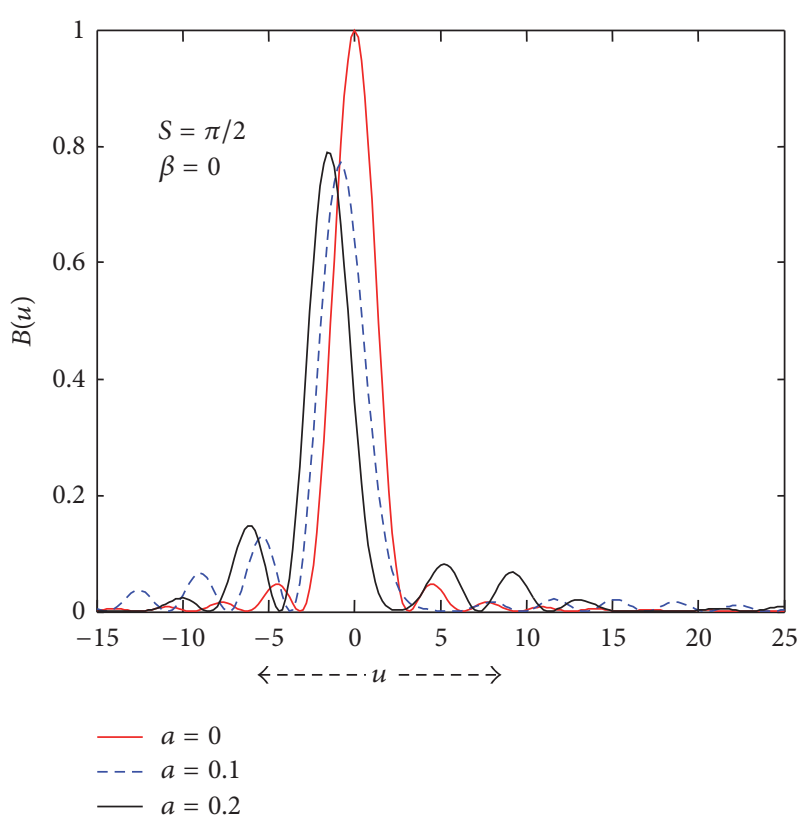

FIGURE 1: Intensity profile for various degree of antiphase apodization when the central region is transparent $(\beta=0)$ for $S=\pi / 2$.

imaging system. The PSF curves are presented in Figure 1 for various degree of asymmetric apodization when the central region of the pupil function is transparent when $S=\pi / 2$. Figure 1 shows that on the left side of the pattern the central maxima is transferred, widened, and lowered at the cost of narrow central peak on the right half side. This effect depends on the width $(a)$ of edge element of the aperture.

The intensity of the first optical side-lobe is decreasing on good side for $b=0.1$ and the dark region has occupied certain distance on good side of the PSF which is the useful effect for imaging of a faint object that is located little away from the bright object in the presence of partial spherical aberration, whereas for $b=0.2$ intensity of lower-order side-lobes is increasing compared to unapodized case. But high-order optical side-lobes completely vanished and the dark region is occurring very closely to the central peak and engaging certain distance can give rise to super resolution for two closely spaced companions with wide variation in their intensities. The PSF profile curves are presented in Figure 2 for various " $a$ " values when the central region of the pupil function is apodized in the presence of partial spherical aberration. Figure 2 depicts that, for $a=0.2$, similar trend results are noticed like Figure 1. But in this case lateral resolution of the central peak is increasing relative to amplitude-only apodized case ( $a$ $=0$ and $\beta=1$ ). It is also observed that lower- and higher-order optical side-lobes completely vanished on both (good and bad) sides for $a=0$ and $\beta=1$. It emphasizes how the amplitude masking is effective in the central region of slit function on the suppression of side-lobes in the diffraction pattern of the optical system under primary spherical aberration. Figure 3 illustrates that in the presence of amplitude-only apodization $(\beta)$ and maximum primary spherical aberration $(S)$ as degree of amplitude apodization $\beta$ increases from 0 to 1 the sidelobes suppression increases on both sides of the central peak.

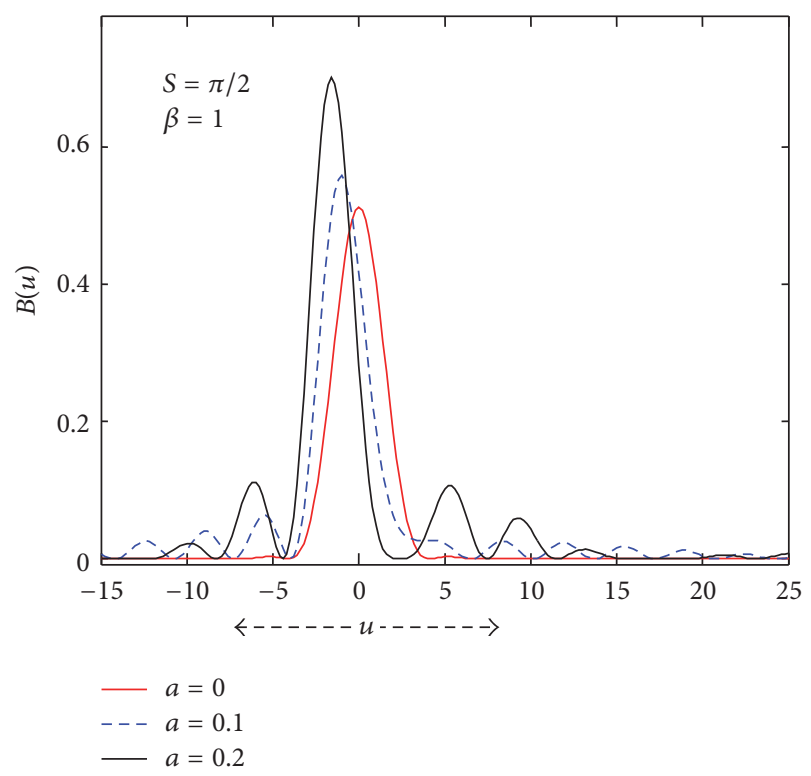

FIGURE 2: Intensity profile for various degree of antiphase apodization when the central region is apodized $(\beta=1)$ for $S=\pi / 2$.

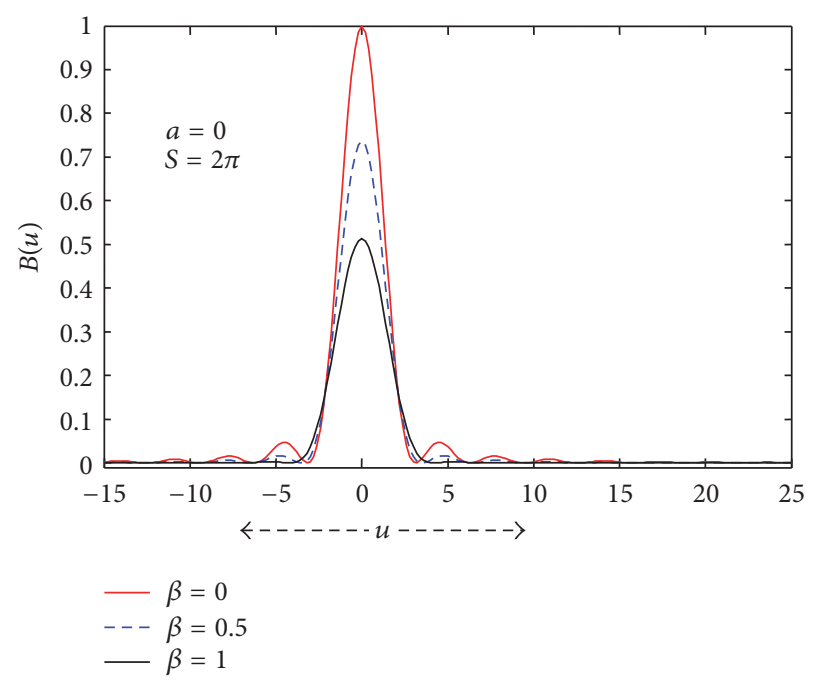

FIGURE 3: Effect of amplitude apodization $(\beta)$ on intensity profile for maximum spherical aberration $(S=2 \pi)$.

In this case the magnitude of optical side-lobe suppression is much larger than the asymmetric-only apodization.

In our study it is also observed that in the presence of optimum antiphase apodization $(a=0.06)$ as amplitude apodization increases the side-lobes suppression increases to a greater extent on good side whereas peak value of main lobe is degrading on the other hand also (on bad side) where a similar trend is noticed. Thus, it can be emphasized that in the presence of maximum primary spherical aberration the optimum values for " $a$ " (phase masking) and $\beta$ (amplitude masking) are 0.06 and 0.5 , correspondingly. These are depicted in Figure 4 in more detail.

Table 1 lists FWHM of point spread function as function of periphery strip width $(a)$ for various values of amplitude 
TABLE 1: FWHM of PSF for all values of $a$ and $\beta$ under influence of spherical aberration.

\begin{tabular}{lccccccccccc}
\hline \multirow{2}{*}{$a$ " (width of fine periphery strip of the pupil) } & \multicolumn{3}{c}{$S=0$ (spherical aberration) } & \multicolumn{3}{c}{$S=\pi$} & \multicolumn{4}{c}{$S=2 \pi$} \\
& $\beta=0$ & $\beta=0.5$ & $\beta=1$ & $\beta=0$ & $\beta=0.5$ & $\beta=1$ & $\beta=0$ & $\beta=0.5$ & $\beta=1$ \\
\hline 0 & 2.7831 & 2.98244 & 3.34106 & 2.7834 & 2.98274 & 3.34132 & 2.7842 & 2.98358 & 3.34208 \\
0.05 & 2.99724 & 3.11808 & 3.27843 & 2.99734 & 3.11817 & 3.2785 & 2.99766 & 3.11844 & 3.27871 \\
0.1 & 2.99787 & 3.00318 & 3.00007 & 2.99789 & 3.0032 & 3.00008 & 2.99792 & 3.00322 & 3.00009 \\
0.2 & 2.9579 & 3.03495 & 3.1144 & 2.9579 & 3.03496 & 3.1144 & 2.95792 & 3.03497 & 3.11441 \\
\hline
\end{tabular}

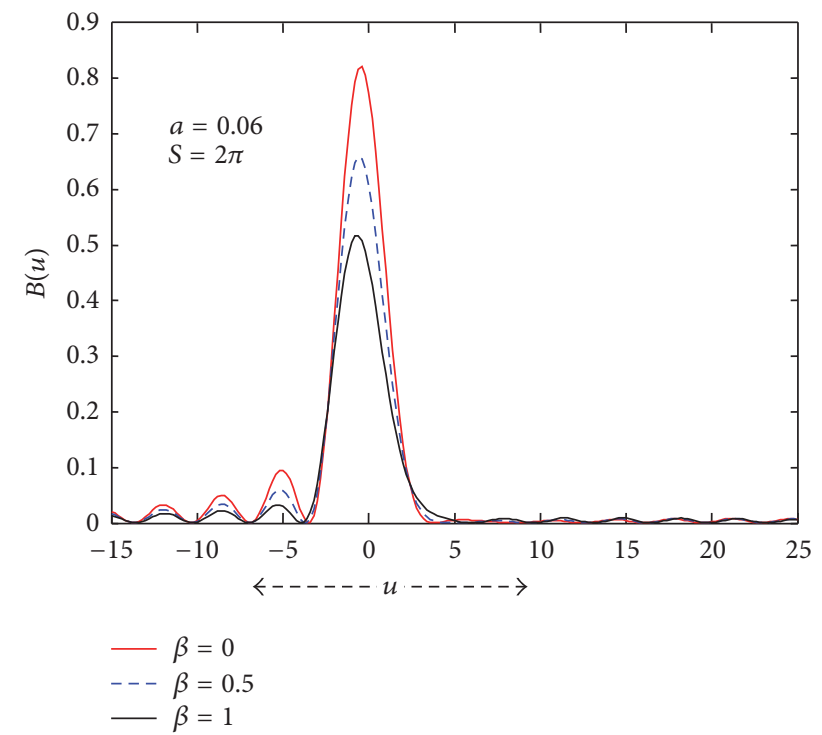

FIGURE 4: Effect of edge strip width (a) and amplitude apodization $(\beta)$ on intensity profile for maximum spherical aberration $(S=2 \pi)$.

masking control parameter $(\beta)$ and spherical aberration $(S)$. These parameter values are obtained for unapodized case, amplitude-only apodized case, phase-only apodized case, and amplitude and phase apodized case. From the listed values in Table 1 for $S=0$ (unapodized case), as " $a$ " increases from 0 to 0.2 the FWHM increases from 2.783 to 2.997 and then decreases to 2.95 with periphery strip width $(a)$. Similar trend is noticed for $S=\pi / 2$ and $S=2 \pi$. It is clear that in the presence of amplitude-only apodization there is improvement in the axial resolution of the spherical aberrated PSF whereas lateral resolution is degrading. On the other hand in the case of phase-only apodization lateral resolution of the central peak is increasing for certain values of " $a$ ". In the presence of spherical aberration for optimum values of antiphase and amplitude masking there is a significant improvement in the axial resolution and lateral resolution of asymmetric PSF. It can be seen in more detail from Table 1 and Figure 4.

\section{Conclusion}

It is concluded that our investigation focused on enhancing the axial and lateral resolution of point spread function under primary spherical aberration, which has been achieved by taking into account the asymmetric pupil mask of onedimensional case. In the presence of primary minimum spherical aberration for $b=0.1$ (phase-only apodization), the intensity of lower-order side-lobes on good side is decreasing and but for $b=0.2$ it is increasing in relation to the unapodized case. On the other hand, high-order optical sidelobes are vanished and the dark region is occurring very closely to the central peak and engaging certain distance can give rise to super resolution for two closely spaced structures with wide variation in their intensities. For $S=\pi / 2$ and $\beta$ $=1$ (amplitude-only apodization) there is a highly effective suppression of the optical side-lobes on both (good and bad) sides of the central peak. Similar observation is found for $S=2 \pi$ and $\beta=0.5$ and 1 . In the presence of any amount of spherical aberration, for transparent central region $(\beta=$ 0 ) FWHM of main peak increases and then decreases with periphery strip width " $a$ " of one-dimensional pupil function. On the whole, it can emphasized that for optimum values of antiphase and amplitude apodization there is a significant improvement in the axial resolution and lateral resolution of aberrated asymmetric point spread function.

\section{Competing Interests}

The authors declare that they have no competing interests.

\section{References}

[1] L. Cheng and G. G. Siu, "Asymmetric apodization," Measurement Science and Technology, vol. 2, no. 3, p. 198, 1991.

[2] G. G. Siu, L. Cheng, D. S. Chiu, and K. S. Chan, "Improved sidelobe suppression in asymmetric apodization," Journal of Physics D: Applied Physics, vol. 27, no. 3, pp. 459-463, 1994.

[3] G. G. Siu, M. Cheng, and L. Cheng, "Asymmetric apodization applied to linear arrays," Journal of Physics D: Applied Physics, vol. 30, no. 5, pp. 787-792, 1997.

[4] A. N. K. Reddy and D. K. Sagar, "Point spread function of optical systems apodized by semicircular array of 2D aperture functions with asymmetric apodization," Journal of Information and Communication Convergence Engineering, vol. 12, no. 2, pp. 8388, 2014.

[5] A. N. K. Reddy and D. Karuna Sagar, "Half-Width at half-Maximum, full-Width at half-Maximum analysis for resolution of asymmetrically apodized optical systems with slit apertures," Pramana, vol. 84, no. 1, pp. 117-126, 2015.

[6] M. Keshavulu Goud, R. Komala, and S. L. Goud, "Point spread function of asymmetrically apodised optical systems with complex pupil filters: the one dimensional case with slit aperture," Acta Physica Polonica A, vol. 122, no. 1, pp. 90-95, 2012.

[7] A. Naresh Kumar Reddy and D. Karuna Sagar, "Two-point resolution of asymmetrically apodized optical systems," Optica Pura y Aplicada, vol. 46, no. 3, pp. 215-222, 2013. 
[8] M. Kowalczyk, C. J. Zapata-Rodríguez, and M. Martínez-Corral, "Asymmetric apodization in confocal scanning systems," Applied Optics, vol. 37, no. 35, pp. 8206-8214, 1998.

[9] W. Yang and A. B. Kotinski, "One-sided achromatic phase apodization for imaging of extrasolar planets," The Astrophysical Journal, vol. 605, no. 2, p. 892, 2004.

[10] M. N. Zervas and D. Tarverner, "Asymmetrically apodized linearly chirped fiber bragg gratings for efficient pulse compression," Fiber and Integrated Optics, vol. 19, no. 4, pp. 355-365, 2000.

[11] I. Escobar, G. Saavedra, M. Martínez-Corral, and J. Lancis, "Reduction of the spherical aberration effect in high-numericalaperture optical scanning instruments," Journal of the Optical Society of America A: Optics and Image Science, and Vision, vol. 23, no. 12, pp. 3150-3155, 2006.

[12] S. Hell, G. Reiner, C. Cremer, and E. H. K. Stelzer, "Aberrations in confocal fluorescence microscopy induced by mismatches in refractive index," Journal of Microscopy, vol. 169, no. 3, pp. 391405, 1993.

[13] G. Saavedra, I. Escobar, R. Martínez-Cuenca, E. SánchezOrtiga, and M. Martínez-Corral, "Reduction of spherical-aberration impact in microscopy by wavefront coding," Optics Express, vol. 17, no. 16, pp. 13810-13818, 2009.

[14] J. H. Mathews and K. D. Fink, Numerical Methods using Matlab, Prentice Hall, New York, NY, USA, 2004. 


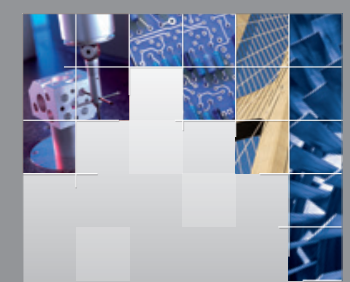

\section{Enfincering}
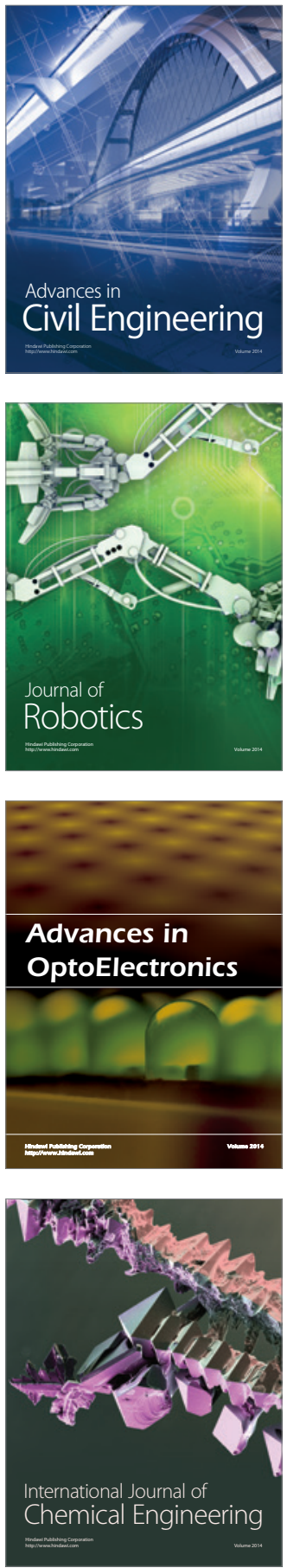

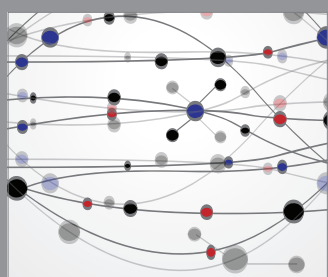

The Scientific World Journal

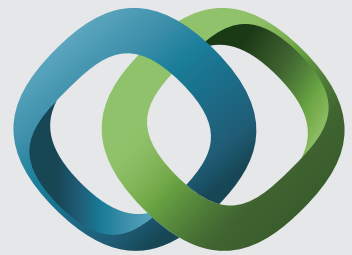

\section{Hindawi}

Submit your manuscripts at

http://www.hindawi.com
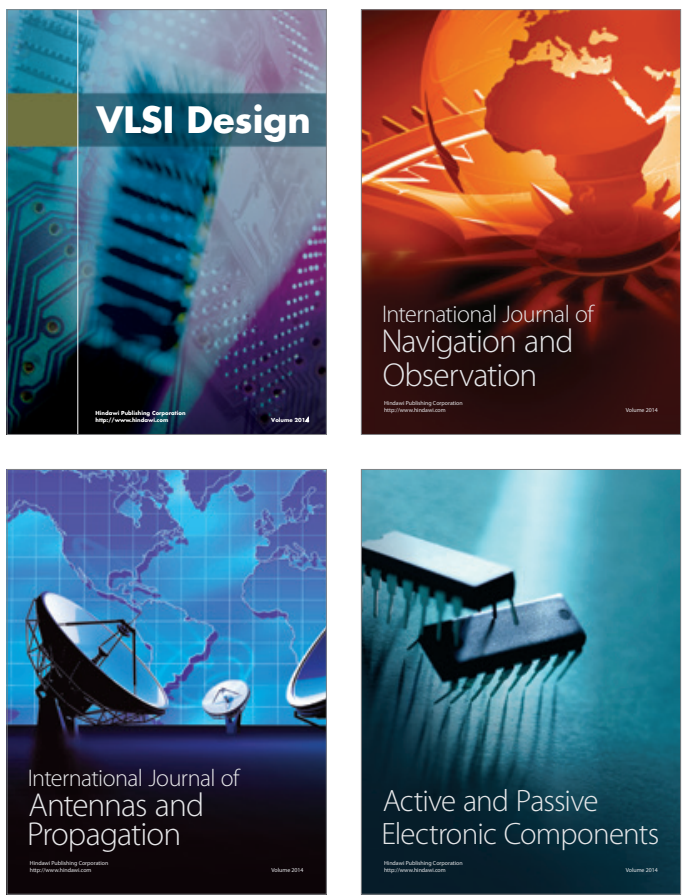
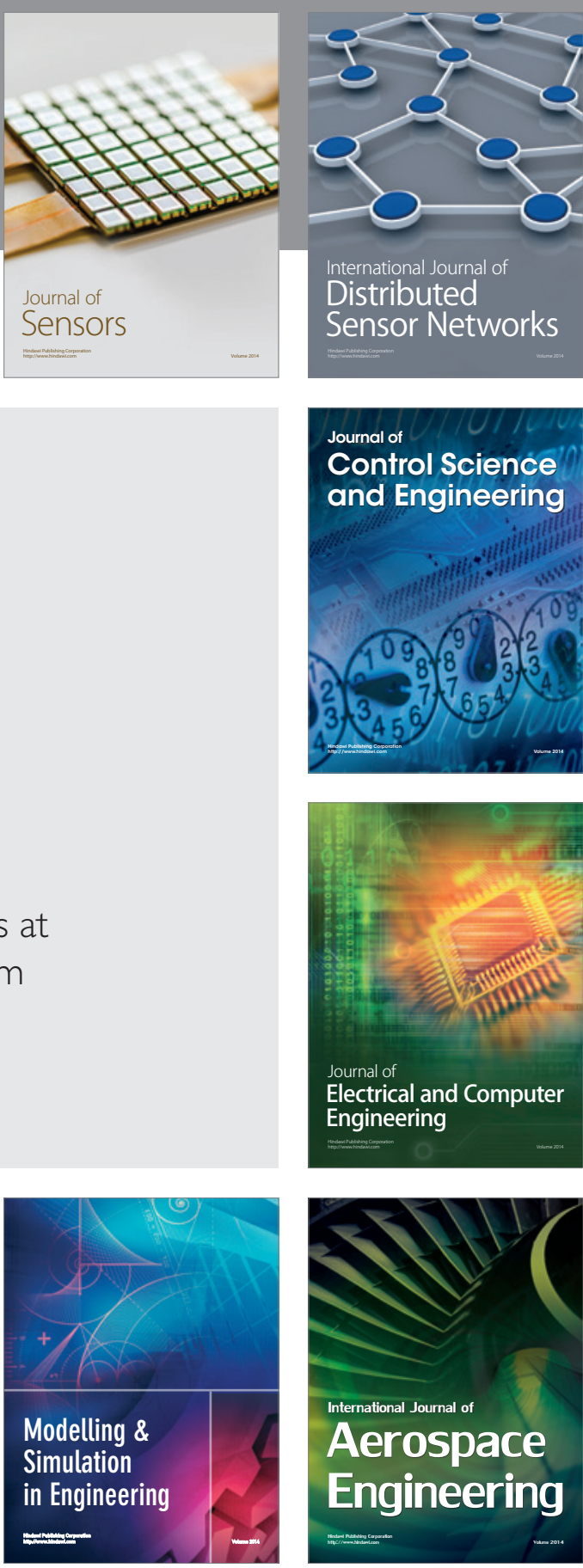

International Journal of

Distributed

Sensor Networks

Journal of

Control Science

and Engineering
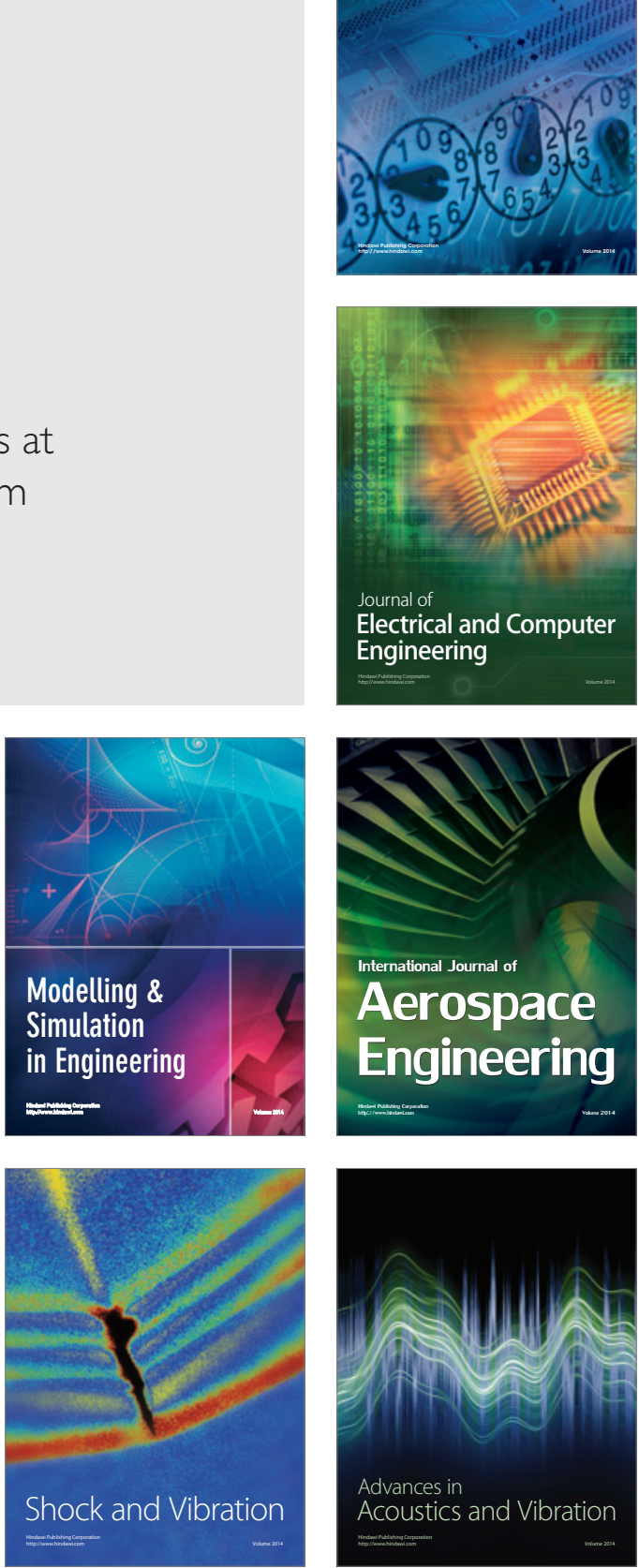